\title{
Cellular Plasticity in Cutaneous Wound Healing
}

\author{
Tang XieLe Liu, ZhiLi Deng and Ji Li* \\ Department of Dermatology, Central South University, China
}

Received: June 07, 2018; Published: June 20, 2018

*Corresponding author: Ji Li, Department of Dermatology, Central South University, China, Tel:8673189753406; Email: liji_xy@csu.edu.cn

\begin{abstract}
Plasticity refers to the capacity of cells to acquire an alternate fate in response to diverse influences. Wound healing is a dynamic and overlapped process including three phases - inflammation, proliferation, and tissue remodeling, involving soluble mediators, blood cells, extracellular matrix, and parenchymal cells. After injury, mature differentiated cells hold within potential to adopt a progenitor-like phenotype (Dedifferentiation) or convert into distinct lineages (Transdifferentiation). Besides, some stem/progenitor cell populations are able to switch into another type, mobilized to repair a wound (Transdetermination). This mini review aims to briefly discuss the phenomenon and mechanisms of the cellular plasticity in cutaneous wound healing for boosting regenerative medicine.
\end{abstract}

Keywords: Cellular plasticity; Reprogramming; Dedifferentiation; Transdifferentiation; Wound Healing

Abbreviations: Th1: T helper 1 Lymphocytes; Th2: T helper 2 Lymphocytes; IFN- $\gamma$ : Interferon- $\gamma$; MFG-E8: Milk Fat Globule Epidermal Growth Factor-8; SD: Sebaceous Duct; IFE: Interfollicular Epidermis; HF: Hair Follicle; SG: Sebaceous Gland; Gata6: Transcription Factor Gata-6; Lgr6: Leucine-Rich Repeat-Containing G-Protein Coupled Receptor 6; Lrig1: Leucine-Rich Repeats and Immunoglobulin-Like Domains Protein 1; Blimp1: PR Domain Zinc Finger Protein 1; ECM: Extracellular Matrix; MSC: Mesenchymal Stem Cells; I.V : Intravenous; GFP: Green Fluorescent Protein; TGF- $\beta$ : Transforming Growth Factor $\beta$; Ang II: Angiotensin II; ET-1: Endothelin-1; P38-MAPK: p38 Mitogen-Activated Protein Kinases; SRF: Serum Response Factor ; TRPC6: Transient Receptor Potential Cation Channel, Subfamily C, Member 6; $\alpha$ SMA: Alpha Smooth Muscle Cell

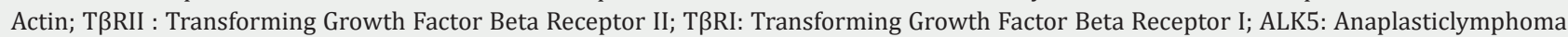
Kinase 5; Smad2/3/4: Mothers Against Decapentaplegic Homolog 2/3/4; SBEs: Bind Smad-Binding Elements; BMP: Bone Morphogenetic Protein

\section{Introduction}

Cellular plasticity is described as the following ways: combination of de-differentiation and re-differentiation, or convert into another lineage directly, which is termed as adaptive cellular reprogramming [1]. Plasticity also occurs in several stem cell populations located in different domains of skin. After injury, stem cells in one compartment are able to differentiate to almost all mature cell types of other compartments [2]. Progenitor cells have the similar property. "Interconversion" is usually used to describe the plasticity of stem cells, whereas "Transdetermination" to progenitor cells [3]. Cutaneous wound healing is characterized by coordinated and overlapping steps including inflammation, proliferation, and remodeling [4]. Cellular plasticity, as a critical physiological healing mechanism, mainly works in the latter two phases.

\section{Cellular Plasticity in Inflammation Phase}

Inflammatory response is crucial to protect the body from invading organisms at wound. The pro-inflammatory macrophages with classical phenotype are named $\mathrm{M}_{1}$ macrophages, corresponding to Th1IFN- $\gamma$-driven responses, while the anti-inflammatory $M_{2}$ macrophages play different roles, corresponding to Th2 responses [5]. Initially, M1 macrophages infiltrate to phagocytose bacteria, foreign debris and dead cells. Hypoxia is an inherent feature of wound, considered to facilitate cell plasticity, holds back the polarization toward to $\mathrm{M}_{1}$ macrophages by decreasing the expression of $\mathrm{T}$ cell costimulatory molecules and chemokine homing receptors $[6,7]$. MFG-E8, generated from inflammatory microenvironments, induces macrophages reprogramming from $M_{1}$ to $M_{2}$ phenotype [8]. Epithelial cells, endothelial cells, smooth muscle cells, pericytes and wound macrophages have been reported as potential local sources releasing MFG-E8 [9]. $M_{2}$ macrophages mainly support cellular proliferation, granulation tissue formation, and angiogenesis in the following phase of wound healing [10].

\section{Cellular Plasticity in Proliferation Phase}

The proliferative stage consists of re-epithelialization of the epidermis and repair of the underlying dermal layer. Recent studies have supposed that the re-epithelialization is modulated by two main cell populations: local epidermal cell at the wound edges and epithelial stem cell from hair follicles or sweat glands [11,12]. With regard to dedifferentiation, the transcription factor Gata6 is the identity of a SD lineage and its downstream transcription factor network controls a lineage switch between sebocytes and SD cells. During wound healing, differentiated Gata6+ cells migrate from the 
SD into the IFE and undergo reversion, acquiring the property to differentiate into a much wider range of epidermal lineages [13]. A Similar example of dedifferentiation is that hair follicles form de novo following wounding in genetically normal adult mice. The nascent follicles arise from cells in the epidermis and/or infundibulum, not the hair follicle stem cell niche,obtaining a phenotype of hair follicle stem cell. The renewed hair was lacking of melanocytes and the regenerated follicles were lacking of bulge-derived epithelial cells, which means related stem cell niche was not re-established $[14,15]$. This wounding-induced renewal could be entirely suppressed by inhibition of Wnt signaling. Correspondingly, the Wnt ligands are over-expressed in the hair follicles near the wound edge [16].

Under homeostatic conditions, the epidermal stem cells in different sites maintain their separate differentiated lineages. However, after injury, different stem cell populations can interconvert functionally. Intriguingly, experimental evidence has supported the idea that stem cells in the IFE can be reprogrammed to HF stem cells on sustained activation of Wnt signaling [17]. The bulge region of the HF has long been supposed as a primary reservoir for epidermal keratinocyte stem cells [18]. Recent lineage tracing experiments revealed that during steady homeostasis, bulge stem cells contribute to HF compartments and the SG. In some adverse conditions, however, these cells rapidly migrate toward the IFE to substitute wounded skin [19]. Additionally, Live cell imaging together with lineage tracing experiments showed that progeny of slow cycling bulge cells (expressing CD34 and K15) migrate into the Lgr6+ Lrig1+ upper bulge region to convert to the Blimp1+ progenitor population and then enter the sebaceous gland as supplement of the mature sebocytes [20].

At the wound site, blood-borne myeloid cells acquire plasticity to transdifferentiate into endothelial cells supporting wound angiogenesis and giving rise to white adipocytes [21,22]. Macrophages are not limited to changing their functional phenotype from pro-inflammatory M1 to anti-inflammatory M2 state, but also the conversion to endothelial cells, endothelial progenitor cells, or endothelial-like cells $[23,24]$. Fibroblasts can be of mesenchymal and myeloid origin like macrophages. Myeloid-converted fibroblast-like cells are the initial contributor of wound site ECM [22]. MSCs accounts for roughly $0.01-0.001 \%$ of the bone marrow derived cell population [25]. The connective tissue sheath and the papilla of the hair follicle probably represent the site for cutaneous MSCs. After i.v. injected with MSCs derived from GFP transgenic mice to wound, GFP-positive cells with specific markers for keratinocytes, endothelial cells, and pericytes could be detected. Accumulating MSCs at wounded sites are able to transdifferentiate into multiple skin cell types, contributing to wound healing [26].

\section{Cellular Plasticity in Remodeling Phase}

Myofibroblasts synthesize ECM components and produce high contraction for epithelial-gap closure or wound remodeling Transdifferentiation from quiescent dermal fibroblasts to secretory and contractile myofibroblasts plays a key role in remodeling and scarring, induced by profibrotic cytokines and chemokines such as TGF- $\beta$, Ang II, connective tissue growth factor, and ET-1 [27]. TGF $\beta$ and/or angiotensin stimulate p38-MAPK-dependent activation of SRF in fibroblasts, which transcriptionally activates the calcium channel TRPC6, allowing calcium entry cells to activate calcineurin, eventually leading to the Trans differentiation [28]. Myofibroblast trans differentiation also involves TGF $\beta$-induced de novo synthesis of $\alpha \mathrm{SMA}+$ fibers. $\alpha \mathrm{SMA}+$ fibers enhance contractility and increase expression of ECM components, like collagen and fibronectin [29]. TGF $\beta 1$ binds to the TGF $\beta$ RII receptor (T $\beta R I I$ ), heterodimerizing with TRRI/ALK5, a complex which can recruit and phosphorylate transcription factors Smad2 and Smad3 [30,31]. Then, Phospho-Smad2/3/4 complexes translocate into the nucleus and SBEs in gene regulatory elements, participating myofibroblast programming [32-34]. Conversion from myofibroblasts to a completely distinct adipocyte lineage also has been reported. The reprogramming requires BMP signaling which triggered by renewed hair follicles, and then activates adipocyte transcription factors which were expressed during development [35].

\section{Conclusion}

Looking at emerging evidences of previous studies provides better understanding on recovery mechanisms. Promoting regenerative medicine by the body's own cell therapy rather than forced genetic tools is likely to become an increasingly significant focus of future work.

\section{References}

1. Jessen KR, Mirsky R, ArthurFarraj P (2015) The Role of Cell Plasticity in Tissue Repair: Adaptive Cellular Reprogramming. Dev Cell 34(6): 613620 .

2. Mascre G, Dekoninck S, Drogat B, Youssef KK, Brohee S, et al. (2012) Distinct contribution of stem and progenitor cells to epidermal maintenance. Nature 489(7415): 257-262.

3. Tata PR, Rajagopal J (2016) Cellular plasticity: 1712 to the present day. Curr Opin Cell Biol 43:46-54.

4. Gurtner GC, Werner S, Barrandon Y, Longaker MT (2008) Wound repair and regeneration. Nature 453(7193): 314-321.

5. Stout RD (2010) Editorial: macrophage functional phenotypes: no alternatives in dermal wound healing? J Leukoc Biol 87(1): 19-21.

6. Raggi F, Pelassa S, Pierobon D, Penco F, Gattorno M, etal. (2017) Regulation of Human Macrophage M1-M2 Polarization Balance by Hypoxia and the Triggering Receptor Expressed on Myeloid Cells-1. Front Immunol 8: 1097.

7. Yoshida Y, Takahashi K, Okita K, Ichisaka T, Yamanaka S (2009)Hypoxia enhances the generation of induced pluripotent stem cells. Cell Stem Cell 5(3): 237-241.

8. Brissette MJ, Laplante P, Qi S, Latour M, Cailhier JF (2016) Milk fat globule epidermal growth factor-8 limits tissue damage through inflammasome modulation during renal injury. J Leukoc Biol 100(5): 1135-1146.

9. Brissette MJ, Lepage S, Lamonde AS, Sirois I, Groleau J, etal. (2012) MFG-E8 released by apoptotic endothelial cells triggers antiinflammatory macrophage reprogramming. Plos One 7(4): 36368.

10. Krzyszczyk P, Schloss R, Palmer A, Berthiaume F (2018) The Role of Macrophages in Acute and Chronic Wound Healing and Interventions to Promote Pro-wound Healing Phenotypes. Front Physiol 9: 419.

11. Lau K, Paus R, Tiede S, Day P, Bayat A (2009) Exploring the role of stem cells in cutaneous wound healing. Exp Dermatol 18(11): 921-933.

12. Roh C, Lyle S (2006) Cutaneous stem cells and wound healing. Pediatr Res 59( 4 pt 2): 100R-103R. 
13. Donati G, Rognoni E, Hiratsuka T, LiakathAli K, Hoste E, et al. (2017) Wounding induces dedifferentiation of epidermal Gata6(+) cells and acquisition of stem cell properties. Nat Cell Biol 19(6): 603-613.

14. Ito M, Yang Z, Andl T, Cui C, Kim N, etal. (2007) Wnt-dependent de novo hair follicle regeneration in adult mouse skin after wounding. Nature 447(7142): 316-320.

15. Nishimura EK, Jordan SA, Oshima H, Yoshida H, Osawa M,etal. (2002) Dominant role of the niche in melanocyte stem-cell fate determination. Nature 416(6883): 854-860.

16. 16. Fathke C, Wilson L, Shah K, Kim B, Hocking A, et al. (2006) Wnt signaling induces epithelial differentiation during cutaneous wound healing. BMC Cell Biol 7: 4.

17. SilvaVargas V, Lo CC, Giangreco A, Ofstad T, Prowse DM, et al. (2005) Beta-catenin and Hedgehog signal strength can specify number and location of hair follicles in adult epidermis without recruitment of bulge stem cells. Dev Cell 9(1): 121-131.

18. Fuchs E, Tumbar T, Guasch G (2004) Socializing with the neighbors: stem cells and their niche. Cell 116(6): 769-778.

19. Nowak JA, Polak L, Pasolli HA, Fuchs E (2008) Hair follicle stem cells are specified and function in early skin morphogenesis. Cell Stem Cell 3(1): 33-43.

20. Petersson M, Brylka H, Kraus A, John S, Rappl G, et al. (2011) TCF/Lef1 activity controls establishment of diverse stem and progenitor cell compartments in mouse epidermis. EMBO J 30(15): 3004-3018.

21. Majka SM, Fox KE, Psilas JC, Helm KM, Childs CR, et al. (2010) De novo generation of white adipocytes from the myeloid lineage via mesenchymal intermediates is age, adipose depot, and gender specific. Proc Natl Acad Sci U S A 107: 14781-14786.

22. Sinha M, Sen CK, Singh K, Das A, Ghatak S, et al. (2018) Direct conversion of injury-site myeloid cells to fibroblast-like cells of granulation tissue. Nat Commun 9(1): 936.

23. Bailey AS, Willenbring H, Jiang S, Anderson DA, Schroeder DA, et al. (2006) Myeloid lineage progenitors give rise to vascular endothelium. Proc Natl Acad Sci USA. 103(35): 13156-13161.

24. Yan D, Wang X, Li D, Qu Z, Ruan Q (2011) Macrophages overexpressing VEGF, transdifferentiate into endothelial-like cells in vitro and in vivo. Biotechnol Lett 33(9): 1751-1758.
25. Pittenger MF, Mackay AM, Beck SC, Jaiswal RK, Douglas R, et al. (1999) Multilineage potential of adult human mesenchymal stem cells. Science 284(5411): 143-147

26. Sasaki M, Abe R, Fujita Y, Ando S, Inokuma D, Shimizu H, et al. (2008) Mesenchymal stem cells are recruited into wounded skin and contribute to wound repair by trans differentiation into multiple skin cell type. J Immunol 180(4): 2581-2587.

27. Hinz B, Phan SH, Thannickal VJ, Prunotto M, Desmouliere A, et al (2012) Recent developments in myofibroblast biology: paradigms for connective tissue remodeling. Am J Pathol 180(4): 1340-1355.

28. Davis J, Burr AR, Davis GF, Birnbaumer L, Molkentin JD (2012) A TRPC6dependent pathway for myofibroblast trans differentiation and wound healing in vivo. Developmental cell 23(4): 705-715

29. Ijaz T, Jamaluddin M, Zhao Y, Zhang Y, Jay J, et al. (2017) Coordinate activities of BRD4 and CDK9 in the transcriptional elongation complex are required for TGFbeta-induced Nox4 expression and myofibroblast transdifferentiation. Cell death \& disease 8(2): e2606.

30. Biernacka A, Dobaczewski M, Frangogiannis NG (2011) TGF-beta signaling in fibrosis. Growth factors (Chur, Switzerland) 29(5): 196-202.

31. Hata A, Chen Y (2016) TGF-beta Signaling from Receptors to Smads. Cold Spring Harbor perspectives in biology 8.

32. Hu B, Wu Z, Phan SH (2003) Smad3 mediates transforming growth factor-beta-induced alpha-smooth muscle actin expression. American journal of respiratory cell and molecular biology 29( 3 pt 1): 397-404.

33. Heldin CH, Miyazono K, ten Dijke P (1997) TGF-beta signalling from cell membrane to nucleus through SMAD proteins. Nature 390(6659): 465471.

34. Evans RA, Tian YC, Steadman R, Phillips AO (2003) TGF-beta1-mediated fibroblast-myofibroblast terminal differentiation-the role of Smad proteins. Experimental cell research 282(2): 90-100.

35. Plikus MV, GuerreroJuarez CF, Ito M, Li YR, Dedhia PH, et al. (2017) Regeneration of fat cells from myofibroblasts during wound healing. Science 355(6326): 748-752.
Submission Link: https://biomedres.us/submit-manuscript.php

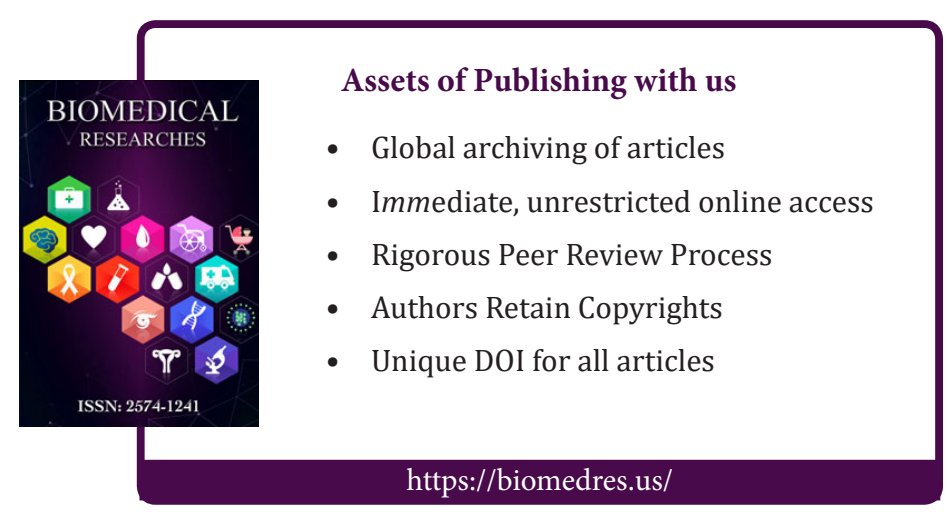

